\title{
Beta-bloqueantes e asma
}

Mariana Baltazar Coelho, ${ }^{1}$ Manuel Branco Ferreira ${ }^{2-3}$

\section{RESUMO}

Introdução: Os doentes asmáticos têm por vezes outras patologias concomitantes para as quais é necessário o uso de beta-bloqueantes. Tendo em conta que a terapêutica com estes fármacos é transversal a diversas doenças e sendo fármacos bastante usados na prática clínica, particularmente nas consultas de cuidados de saúde primários, torna-se importante esclarecer possíveis contraindicações ao seu uso nos indivíduos com asma.

Objetivo: Avaliar os efeitos que a administração de beta-bloqueantes cardioseletivos e não seletivos podem ter em doentes asmáticos no que diz respeito a: alterações nas provas de função respiratória; exacerbações agudas da doença e sua sintomatologia associada; frequência de uso de medicação de alívio; necessidade de recorrer a um serviço de urgência e frequência de internamentos relacionados com efeitos adversos da toma do fármaco.

Métodos: Foi realizada uma revisão sistemática da literatura, tendo sido utilizada a base de dados eletrónica PubMed. A expressão de busca utilizada foi: asthma AND beta-blockers.

Resultados: A pesquisa inicial levou à obtenção de uma amostra de 151 artigos, dos quais 49 foram selecionados pela leitura do título e resumo. Destes, depois da leitura integral e da aplicação dos critérios de inclusão, foram escolhidos 30 artigos. 0 atenolol e o propranolol foram os beta-bloqueantes mais estudados. Dois ensaios clínicos randomizados e um coorte abordaram beta-bloqueantes oculares tópicos; 22 ensaios clínicos randomizados e cinco coortes estudaram beta-bloqueantes orais ou IV.

Conclusões: Os doentes com asma ligeira a moderada poderão, se indicado, pela existência de outras patologias, ser medicados com beta-bloqueante, sempre com vigilância apertada do doente, especialmente nas fases iniciais desta terapêutica. Não se verificou que os beta-bloqueantes cardioseletivos sejam claramente mais seguros que os não seletivos, pelo que terão de ter os mesmos cuidados na sua utilização em asmáticos. Os beta-bloqueantes tópicos oculares podem ter efeitos prejudiciais nos asmáticos, reduzindo o FEV1 e causando exacerbações de asma. Ainda não existem dados suficientes para recomendar o uso de beta-bloqueantes para o tratamento da asma.

Palavras-chave: Asma; Beta-bloqueante; FEV1; Broncoconstrição; Sibilância.

\section{INTRODUÇÃO}

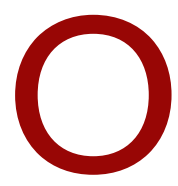

s beta-bloqueantes são utilizados em diversas patologias como a angina, enfarte agudo de miocárdio, insuficiência cardíaca, hipertensão arterial, arritmias e tirotoxicose, bem como para reduzir complicações no período perioperatório. ${ }^{1}$ Os asmáticos podem apresentar estas condições concomitantemente à sua asma, o que levanta a questão de usar ou não um beta-bloqueante nestes doentes e que características o mesmo deve possuir para que não sejam induzidos efeitos respiratórios adversos.

Estes fármacos apresentam diversas propriedades

1. Médica Interna de Medicina Geral e Familiar. USF Costa do Estoril, ACeS Cascais. Estoril, Portugal.

2. Serviço de Imunoalergologia, Centro Hospitalar Universitário Lisboa Norte. Lisboa, Portugal.

3. Clínica Universitária de Imunoalergologia, Faculdade de Medicina da Universidade de Lisboa. Lisboa, Portugal. individuais: a seletividade ou não para o adrenorecetor beta 1 , atividade agonista parcial ou simpaticomimética intrínseca e ações vasodilatadoras, tanto pelo bloqueio dos adrenorecetores vasculares alfa como pela libertação de óxido nítrico. ${ }^{2-3}$

As recomendações para o seu uso na asma foram variando ao longo dos anos, sendo que em 2004 as Guidelines da European Society of Cardiology (ESC) apresentavam a asma como uma contraindicação para o uso de qualquer tipo de beta-bloqueantes, devido ao aumento da resistência nas vias aéreas que podia pôr em risco a vida. Referiam ainda que estas contraindicações podiam ser relativas em doentes em que o benefício da terapia podia suplantar os riscos dos efeitos secundários. Já nas de 2016, também da ESC, ${ }^{4}$ é mencionado que quando um beta-bloqueante cardioseletivo é indicado, a asma não é necessariamente uma 
contraindicação, mas a prescrição desse fármaco deve ser vigiada e acompanhada pelo profissional de saúde.

É neste contexto, e pela necessidade de reunir os dados presentes na literatura que definam particularidades no uso de beta-bloqueantes nestes doentes, que surgiu a presente revisão sistemática cujo objetivo é avaliar os efeitos que a administração de beta-bloqueantes cardioseletivos e não seletivos, tanto por via oral, intravenosa ou tópica ocular, podem ter em doentes asmáticos no que diz respeito a alterações da função pulmonar, exacerbações da doença e sintomatologia associada, frequência de uso de medicação de alívio, recurso a serviço de urgência e frequência de internamentos relacionados com a toma do fármaco.

\section{MÉTODOS}

Esta revisão sistemática foi construída tendo por base a recomendação PRISMA. ${ }^{5}$

\section{Critérios de seleção de estudos}

Para a elaboração da questão de investigação, definição dos critérios de inclusão e de exclusão foram definidas as características do estudo usando a estratégia PICOS, a saber:

- P: Participantes - Indivíduos adultos (mais de 18 anos) com diagnóstico de asma.

- I: Intervenção - Exposição a beta-bloqueantes cardioseletivos e não seletivos, com doses únicas, múltiplas ou contínuas, e administração IV, oral ou tópica.

- C: Comparador-Comparações entre resultados das variáveis dos estudos.

- O: Resultados - Alterações na função pulmonar avaliadas nas provas de função respiratória, sibilância ou sintomas de exacerbação da asma, uso de terapêutica de alívio, número de idas à urgência e frequência de internamentos hospitalares.

- S: Estudos - Estudos cruzados, controlados por placebo, randomizados e cegos e estudos coorte.

Os estudos deveriam ter os métodos utilizados explícitos e com uma amostra de doentes asmáticos adultos (18 anos ou mais). Para além disto, estabeleceu-se como critério de inclusão o intervalo de tempo entre 1981 e 2017 para a pesquisa (para se abranger uma grande quantidade de estudos e serem mais recentes, logo, com conteúdos à partida mais atualizados), bem como a disponibilidade dos textos na sua versão completa.

\section{Métodos de pesquisa}

A revisão de literatura realizou-se entre fevereiro de 2017 e setembro de 2017.

A pesquisa foi feita no idioma inglês na plataforma PubMed.

A expressão de busca utilizada foi: asthma AND beta-blockers.

\section{Seleção dos estudos}

O processo de seleção dos estudos e respetiva avaliação dos mesmos, através da aplicação dos critérios de inclusão e de exclusão, foi feita em paralelo por dois investigadores.

Primeiramente foram analisados os títulos e abstracts dos artigos encontrados que cumpriam os critérios de seleção estabelecidos. De seguida, após a obtenção da versão completa dos artigos selecionados na primeira fase de pesquisa procedeu-se à sua leitura na íntegra, de modo a incluir os que seguiam os critérios de inclusão.

\section{Procedimento de recolha de dados}

Os dados dos estudos selecionados foram trabalhados de modo a poder obter o tema do estudo, o tipo de estudo, a duração do estudo, as características da amostra estudada (dimensão e idade dos participantes, bem como algumas características particulares dos mesmos), as intervenções realizadas, os resultados medidos e as evidências do estudo.

\section{Avaliação do risco de viés nos estudos incluídos}

Foram incluídos apenas estudos que apresentassem os critérios de inclusão de forma explícita, que tivessem uma boa descrição relativamente à amostra tratada bem como do tipo de estudo e ainda que revelassem uma boa descrição da metodologia usada.

Poder-se-á considerar um viés o facto de terem sido selecionados artigos apenas na língua inglesa, sendo questionável se será legítimo aplicar as conclusões deste estudo à realidade portuguesa.

\section{RESULTADOS}

\section{A. Seleção dos estudos}

A amostra potencial era constituída por 151 artigos em resultado da pesquisa na PubMed. A leitura e a análise dos títulos e abstracts permitiram excluir parte destes artigos por não cumprirem os critérios de inclusão. 


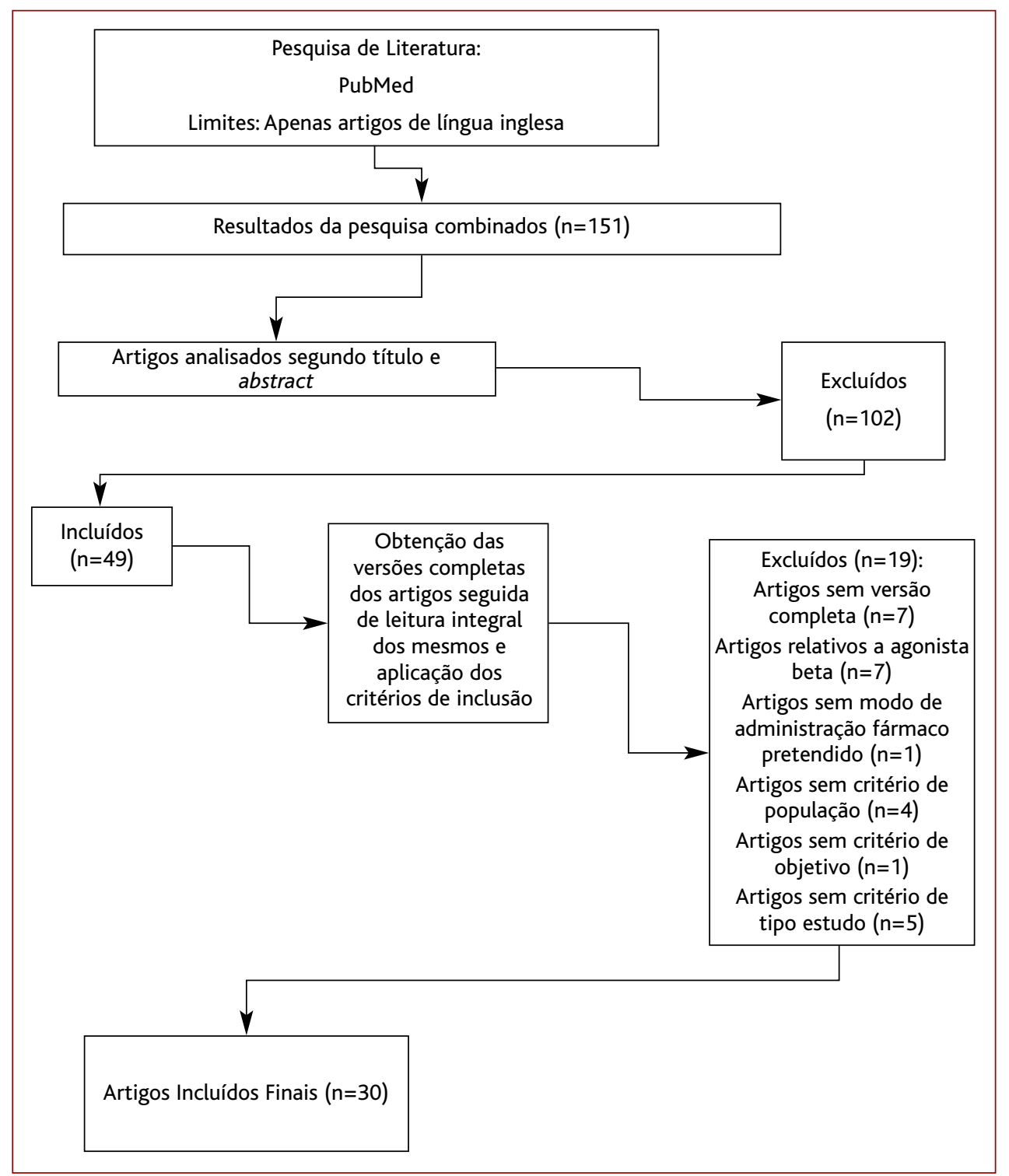

Figura 1. Diagrama de fluxo na seleção de artigos.

Assim, selecionaram-se 49 artigos que pareciam poder incluir-se nos critérios previamente definidos.

Desta amostra apenas se conseguiu obter a versão completa de 42 artigos.

Após a leitura das versões completas de cada artigo foram retirados quatro destes 42 artigos por não cumprirem os critérios de inclusão. Assim sendo, restaram 38 artigos.

Excluíram-se posteriormente mais oito artigos por diversas razões identificadas, obtendo-se assim 30 artigos finais para este estudo (Figura 1). As principais características e resultados de cada estudo encontram-se sumarizadas no Material Suplementar. ${ }^{6-35}$

Dos 30 artigos finais identificaram-se seis estudos coorte (de tipo observacional sem intervenção) e 24 ensaios clínicos randomizados. Destes 30 estudos três avaliaram os efeitos de beta-bloqueantes administrados por via tópica ocular. 


\section{B. Características dos estudos e outcomes avaliados}

A idade média de todos os participantes dos estudos incluídos foi de 43,5 anos.

A Tabela 1 representa os beta-bloqueantes estudados e o número de estudos em que foram incluídos, sendo o atenolol e o propranolol os beta-bloqueantes mais frequentemente estudados.

Relativamente aos outcomes avaliados em cada estudo, 25 artigos incluíram valores das provas de função pulmonar, dos quais os parâmetros mais frequentemente estudados foram: FEV1 em 24 estudos; FVC em 11 estudos; PEFR em 10 estudos e o sGaw em quatro estudos. Outros outcomes avaliados foram: medicação de alívio para a exacerbação da asma (cinco artigos), presença de sibilância (dois artigos), idas ao serviço de urgência (dois artigos), frequência de internamentos hospitalares (três artigos) e mortalidade (dois artigos).

Por fim, foram ainda analisados outros parâmetros apenas num único estudo com: frequência de exacerbações de asma, questionário de controlo de asma, a taxa de reinternamento em seis meses, avaliação analítica de hemograma, função urinária e bioquímica, curvas dose-resposta de fármacos, scores de sintomatologia e quantificação dos dias sem exacerbações de asma.

\section{Tipos e resultados dos estudos}

\section{1) Ensaios clínicos randomizados:}

Foram identificados 22 ensaios clínicos randomizados, oito dos quais avaliaram a exposição a ambos os tipos de beta-bloqueantes; ${ }^{7,17-18,20,28,31,33-34}$ cinco avaliaram apenas a exposição a não seletivos ${ }^{11,15,26,30,35}$ e nove apenas a cardioseletivos. ${ }^{14,19,22-25,27,29,32}$

A Tabela 2 representa o tipo de administração das doses e a seletividade do beta-bloqueante estudado e o número de estudos correspondentes.

C. 1.1) Ensaios que incluíram avaliação de doses únicas: ${ }^{7,14-15,17-20,22-27,29-35}$

Foram analisados um total de 273 doentes com idade média de 39,8 anos. Todos os ensaios com doses únicas apenas avaliaram como outcome as provas de função respiratória, sendo o FEV1 o parâmetro mais frequentemente estudado.

\section{1.1.1) Doses únicas de beta-bloqueantes cardioseleti-} vos:

\begin{tabular}{|c|c|}
\hline Beta bloqueantes seletivos & Beta bloqueantes não seletivos \\
\hline Atenolol - 11 & Propranolol - 7 \\
\hline Metoprolol - 6 & Pindolol - 5 \\
\hline Celiprolol - 4 & Timolol - 4 ( 1 em tópico ocular) \\
\hline Bisoprolol - 4 & Labetalol - 3 \\
\hline Esmolol - 2 & Nadolol - 2 \\
\hline Bevantolol - 2 & Sotalol - 2 \\
\hline Betaxolol - 2 & Dilevalol - 2 \\
\hline Nebivolol - 1 & Carvedilol - 2 \\
\hline Xameterol - 1 & $( \pm)$-sotalol - 1 \\
\hline IC $141,292-1$ & L-414,465 - 1 (tópico ocular) \\
\hline \multicolumn{2}{|l|}{ Landiolol - 1} \\
\hline \multicolumn{2}{|l|}{ Acebutolol - 1} \\
\hline BPPA - 1 (tópico ocular) & \\
\hline
\end{tabular}

Nos 16 ensaios em que foram analisadas doses únicas de beta-bloqueantes cardioseletivos registaram-se 28 exposições a diferentes fármacos, das quais 15 $(53,6 \%)$ mostraram uma diminuição do FEV1 estatisticamente significativa relativamente ao seu valor inicial e 12 das 28 exposições $(42,9 \%)$ revelaram alterações semelhantes às do placebo. ${ }^{7,14,17-20,22-25,27,29,31-34}$ Apenas uma das exposições (3,6\%) (celiprolol 400mg) se associou a um aumento do FEV1 considerado estatisticamente significativo. Nos ensaios que apresentaram diminuição do FEV1 constatou-se um decréscimo deste valor entre $0,1 \%$ (valor mínimo) e $15,9 \%$ (valor máximo) e entre 0,025 l (valor mínimo) e 1,07 l (valor máximo). A média ponderada de diminuição do FEV1 foi de 8,24\% e 0,241 .

C. 1.1.2) Doses únicas de beta-bloqueantes não seletivos:

Nos 11 ensaios em que foram analisadas doses únicas de beta-bloqueantes não seletivos registaram-se 17 exposições a diferentes fármacos, das quais seis $(35,3 \%)$ revelaram uma diminuição estatisticamente significativa do FEV1 em relação aos valores no início do estudo e nove exposições (52,9\%) não revelaram alterações significativas. $7,15,17-18,20,26,30-31,33-35$ As outras duas exposições $(11,8 \%)$ ocorreram num ensaio ${ }^{30}$ que não avaliou o FEV1, mas outros parâmetros. Nos ensaios 


\begin{tabular}{|c|c|c|c|c|c|}
\hline & $\begin{array}{l}\text { Apenas uma } \\
\text { dose } \\
n=15\end{array}$ & $\begin{array}{l}\text { Várias doses } \\
\text { dadas em } \\
1 \text { ou } 2 \text { dias } \\
n=1\end{array}$ & $\begin{array}{l}\text { Combinação de } \\
\text { doses únicas e } \\
\text { várias doses } \\
n=1\end{array}$ & $\begin{array}{l}\text { Doses contínuas administradas } \\
\text { cronicamente ao longo do } \\
\text { tempo } \\
n=1\end{array}$ & $\begin{array}{l}\text { Combinação doses } \\
\text { únicas e doses } \\
\text { contínuas } \\
n=4\end{array}$ \\
\hline $\begin{array}{l}\text { Beta-bloqueantes } \\
\text { não seletivos }\end{array}$ & $n=3^{15,30,35}$ & & & $n=1^{11}$ & $n=1^{26}$ \\
\hline $\begin{array}{l}\text { Beta-bloqueantes } \\
\text { cardioseletivos }\end{array}$ & $n=6^{14,23-25,27,29}$ & & $n=1^{22}$ & & $n=2^{19,32}$ \\
\hline $\begin{array}{l}\text { Beta-bloqueantes } \\
\text { não seletivos } \\
+ \\
\text { Beta-bloqueantes } \\
\text { cardioseletivos }\end{array}$ & $n=6^{17-18,20,31,33-34}$ & $n=1^{28}$ & & & $n=1^{7}$ \\
\hline
\end{tabular}

que apresentaram diminuição do FEV1 constatou-se uma redução deste valor entre 1,5\% (valor mínimo) e $17 \%$ (valor máximo) e entre de 0,01 1 (valor mínimo) e 0,261 (valor máximo). A média ponderada de diminuição do FEV1 foi de 6,71\% e 0,09 l.

C. 1.2) Ensaios com doses múltiplas (administradas sucessivamente em intervalos de tempo definidos, em um ou dois dias de estudo):22,28

Nestes dois ensaios foram seguidos apenas 10 doentes, com uma média de 41,5 anos de idade. Num dos ensaios, a primeira fase do estudo incluiu apenas dois doentes e testou a administração de doses sucessivamente maiores do beta-bloqueante cardioseletivo ICI 141,292 (25, 50, 100 e 200mg), em intervalos de duas horas durante um dia. ${ }^{22}$ Verificou-se, com a dose máxima de 200mg, uma diminuição do FEV1 de 9\% num dos doentes e de $4 \%$ no outro. O segundo ensaio, com oito doentes, revelou a ocorrência de sibilância em quatro doentes, que se seguiu à toma de doses de 10, 17,5 e $45 \mathrm{mg}$ de propranolol e de $37,5 \mathrm{mg}$ de metoprolol. ${ }^{28} \mathrm{Os}$ restantes quatro doentes toleraram todas as doses cumulativas administradas.

C. 1.3) Ensaios com doses contínuas (administradas cronicamente ao longo do tempo): $:$,11,19,26,32

Nos cinco ensaios clínicos, com duração variável de três a 12 semanas, foi incluído um total de 93 doentes com idade média de 47,1 anos. Os ensaios com doses contínuas avaliaram não só provas de função respiratória como também o recurso a medicação de alívio, scores de sintomas e presença de sibilância, avaliando, assim, também o controlo da asma nos doentes.

C. 1.3.1) Doses contínuas de beta-bloqueantes cardioseletivos: ${ }^{7,19,32}$

Dois ensaios, com um total de 28 doentes, não apresentaram alterações no controlo da asma. ${ }^{7,19}$ No entanto, um outro, com 14 doentes, mostrou, em comparação com placebo, valores significativamente diferentes da frequência de exacerbações de asma (298 vs 272) e episódios de sibilância (74 vs 60), menor número de dias livres de doença (199 vs 217) e alterações nos valores do peak flow, tanto dos matinais como dos vespertinos (325,5 vs 341,9 e 335,5 vs 341,9, respetivamente) com o metoprolol..$^{32}$ Quanto aos valores de FEV1 não se verificaram quaisquer alterações estatisticamente significativas, tanto com o atenolol como com o metoprolol.

C.1.3.2) Doses contínuas de beta-bloqueantes não seletivos: ${ }^{7,11,26}$

A exposição ao propranolol em associação com tiotrópio, ${ }^{7}$ avaliada em 16 doentes, não revelou qualquer alteração no controlo asma e não apresentou diferenças significativas no FEV1 relativamente aos seus valores iniciais. Um outro ensaio, com 41 doentes avaliando a exposição ao labetalol, também não revelou diferenças estatisticamente significativas no FEV1 ao longo das três semanas de estudo. ${ }^{26}$

Apenas um ensaio com 10 doentes mostrou uma diminuição estatisticamente significativa do FEV1, da 
ordem dos $5 \%$, com a dose escalada de nadolol, embora não tenham ocorrido mudanças significativas no controlo da asma, PEFR ou uso de medicação de alívio. ${ }^{11}$ C. 2) Estudos coorte (estudos de tipo observacionais sem intervenção): $:, 8,10,12-13$

Os cinco estudos com duração entre oito meses e 11 anos envolveram doentes com idade média de 61 anos e englobaram um total de 48.796 doentes. Dois estudos englobaram doentes com asma e/ou DPOC. ${ }^{12-13}$ Os restantes três estudos avaliaram exclusivamente doentes asmáticos. ${ }^{6,8,10}$

Foram avaliados mais frequentemente outros outcomes que não as provas de função respiratória, como a presença de sibilos na auscultação, ${ }^{10}$ mortalidade num ano, ${ }^{13}$ readmissões hospitalares em seis meses ${ }^{13}$ ou exacerbações moderadas e/ou graves, ${ }^{6,8,12}$ sendo a prescrição de corticoides orais e as idas à urgência englobadas no grupo de exacerbações moderadas e os internamentos e morte pela doença no grupo de exacerbações graves.

C. 2.1) Beta-bloqueantes cardioseletivos e não seletivos analisados isoladamente: ${ }^{:, 10,12}$

Num dos estudos, com 35.502 doentes com asma e doença cardiovascular, os beta-bloqueantes cardioseletivos não levaram a risco significativo de exacerbações de asma moderadas (IRR $=0,97$, IC95\%, 0,85-1,11, $p=0,658$ ) ou graves (IRR $=0,87$, IC5\%, $0,57-1,35, p=0,540$ ), independentemente da dose ou duração do uso e os beta-bloqueantes não-seletivos aumentaram o risco de exacerbação moderada da asma, mesmo quando iniciados com doses médias ou baixas (IRR=5,16, IC95\%, 1,83-14,54, $p=0,002)$ ou de exacerbação tanto moderada (IRR=2,67, IC95\%, 1,08-6,62, $p=0,034$ ) como grave (IRR=12,11, IC95\%, 1,02-144,11, $p=0,048$ ), quando usados cronicamente em altas doses. ${ }^{6}$

Um outro estudo com esmolol e landiolol, que incluiu 60 doentes, mostrou que estes fármacos podem ser usados com segurança no perioperatório de doentes com hiperreatividade das vias aéreas (não ocorreu diferença significativa na incidência de sibilância durante a indução da anestesia, a qual ocorreu em cinco doentes do grupo controlo, seis do grupo esmolol e quatro do grupo landiolol). ${ }^{10}$

Contudo, num outro estudo, que envolveu 11.592 doentes com asma e/ou DPOC, verificou-se o aumento de internamentos e idas à urgência quer com os beta- bloqueantes cardioseletivos (risco relativo combinado de 1,34, IC95\%, 1,16-1,55) quer com os não seletivos (risco relativo combinado 1,34, IC95\%, 1,04-1,74). ${ }^{12}$

C.2.3) Beta-bloqueantes cardioseletivos e não seletivos avaliados agrupadamente: $: 13$

Um estudo com 1.527 doentes, que tinha como objetivo determinar a possível associação de uma nova prescrição de beta-bloqueante oral com exacerbações graves de asma, não revelou nenhuma diferença estatisticamente significativa na proporção de doentes a quem foram prescritos corticoides orais nas semanas que se seguiram à nova prescrição de beta-bloqueante, relativamente ao número de doentes a quem tinham sido prescritos corticoides orais de alívio antes do início do estudo: os corticoides orais foram prescritos a $0,8 \%$ dos doentes nas primeiras duas semanas (IRR=0,87, IC95\%, 0,25-2,99); 0,8\% nas segundas duas semanas (IRR=0,89, IC95\%, 0,26-2,97) e $0,8 \%$ nas semanas quatro a oito (IRR=0,80, IC95\%, $0,39-1,64) .{ }^{8}$

$\mathrm{O}$ outro estudo com 115 doentes, que avaliou num mesmo grupo doentes com DPOC ou asma pós enfarte agudo de miocárdio, que teriam iniciado por este motivo terapêutica com beta-bloqueantes, analisou a mortalidade num ano e a frequência de readmissões hospitalares em seis meses por exacerbação de DPOC ou asma. ${ }^{13} \mathrm{O}$ uso de beta-bloqueantes em doentes com DPOC ou asma de gravidade ligeira foi associado a um decréscimo da mortalidade num ano ( $R R=0,88$, IC95\%, 0,69-1,14), semelhante aos indivíduos sem doença pulmonar. Não houve qualquer aumento significativo da frequência de readmissões hospitalares por asma ou DPOC tanto para doentes com doença de gravidade ligeira (RR=0,75, IC95\%, 0,49-1,16) como grave (RR=0,76, IC95\%, 0,51-1,16), comparativamente também aos indivíduos sem doença pulmonar.

Um outro aspeto da terapêutica beta-bloqueante prende-se com a utilização destes fármacos por via tópica ocular para o tratamento do glaucoma. Relativamente a este tema foram incluídos três estudos referentes ao uso de beta-bloqueantes oculares tópicos em doentes asmáticos: um coorte ${ }^{9} \mathrm{e}$ dois ensaios clínicos randomizados duplo-cegos. ${ }^{16,21}$

O coorte com 693 doentes, que avaliou os agentes timolol (não seletivo) e betaxolol (cardioseletivo) em doentes com asma e DPOC estudados num grupo conjunto, concluiu que, em relação a outros fármacos não 
beta-bloqueantes no tratamento crónico tópico antiglaucoma, tanto o timolol como o betaxolol aumentaram os dias de internamento hospitalar (em 20,7 dias e 23,1 dias, respetivamente, por 100 anos de tratamento antiglaucoma) e a frequência de idas ao serviço de urgência (em 6,1 e 7,3 idas, respetivamente, por 100 anos de terapêutica). ${ }^{9}$

Um dos ensaios clínicos randomizado estudou a administração de altas concentrações de BPPA (butilamino-fenoxi-propanol-acetato - um beta-bloqueante tópico oculoseletivo usado para reduzir a pressão intraocular), timolol e placebo em 12 doentes asmáticos com pressão intraocular normal. Ocorreram reduções dose dependentes no FEV1 (média de 23,33\%) e no sGaw (média de 55,41\%), bem como sintomas respiratórios adversos em todos os doentes (taquipneia, dispneia e/ou tosse) com o uso de timolol, mas não ocorreram tais alterações com o BPPA, sugerindo-se, assim, que este agente poderá ter maior margem de segurança em relação ao timolol. ${ }^{16}$

O outro ensaio clínico randomizado envolveu 10 doentes que tinham broncoconstrição com gotas oculares de timolol e testou a exposição a gotas oculares de L-414,465 (R-enantiómero do timolol), o qual também mostrou reduzir de forma dose dependente o sGaw e FEV1, embora quatro vezes menos que o timolol. ${ }^{21}$ Apesar desta diferença, os autores concluem que o L-414,465 não tem maior margem de segurança que $o$ timolol e não tem, portanto, maior vantagem no seu uso clínico.

\section{DISCUSSÃO}

A ideia original de que os beta-bloqueantes poderiam ter efeitos adversos pulmonares foi baseada em casos clínicos que descreviam doentes que tinham broncoconstrição aguda precipitada por altas doses de beta-bloqueantes não seletivos, o que se relacionava com o seu bloqueio nos recetores beta 2 no músculo liso brônquico. ${ }^{44-47}$

Quanto aos beta-bloqueantes não seletivos, resultados de 16 ensaios anteriores a 2002, mostraram que o uso regular de beta-bloqueantes não seletivos comparado com placebo associava-se a diminuição de 13,5\% no FEV1, não se tendo, contudo, verificado nenhum aumento significativo nos sintomas ou no uso de inaladores de alívio. ${ }^{48}$ Neste estudo verificou-se ainda um decréscimo de 22,5\% na resposta broncodilatadora ao agonista beta 2 , sendo que este facto poderia ser visto como um maior risco de efeito adverso clinicamente significativo durante uma exacerbação da asma. ${ }^{48}$

Em 2014, uma revisão sistemática com meta-análise, que apenas avaliava o efeito da exposição aguda a beta-bloqueantes, demonstra que o beta bloqueio agudo não seletivo nas doses dadas causava diminuições não significativas no FEV1, aumento significativo nos sintomas e marcada redução de resposta ao agonista beta 2 inalado. ${ }^{50}$

Em 2014, outro estudo recomenda que sejam evitados os beta-bloqueantes não seletivos porque, apesar de alguns doentes com asma conseguirem tolerar a sua exposição aguda, o risco é maior e a terapêutica de alívio é menos efetiva, sugerindo que o seu risco provavelmente pesa mais que os seus eventuais benefícios. ${ }^{51}$

Na presente revisão sistemática constatou-se que os beta-bloqueantes não-seletivos em doses únicas causaram reduções do FEV1 estatisticamente significativas em $40 \%$ das 15 exposições totais, com uma diminuição média de 6,71\% e 0,09 l. Em relação às doses contínuas, apenas um dos três ensaios considerados revelou diminuições significativas no FEV 1 que, ainda assim, foram reduções ligeiras (5\%) e nenhum dos ensaios mostrou alterações no controlo da asma. Contudo, as duas coortes revelaram resultados desfavoráveis ao uso dos beta-bloqueantes não seletivos, mostrando que estes fármacos podem aumentar o risco de exacerbação de asma tanto moderada como grave.

Relativamente aos beta-bloqueantes cardioseletivos, numa meta-análise realizada em 2002 foi estudado o efeito destes fármacos em doentes com asma ou DPOC com uma componente obstrutiva reversível. ${ }^{48} \mathrm{O}$ estudo avaliou beta-bloqueantes administrados tanto em dose única como num período de três dias a quatro semanas. Dos 80 ensaios incluídos, nenhum demonstrou um aumento nos sintomas respiratórios para os betabloqueantes cardioseletivos comparado com placebo ou com os valores iniciais. Foi relatado que os beta-bloqueantes cardioseletivos sem atividade simpaticomimética intrínseca apenas apresentavam um decréscimo mínimo no FEV1, associado à primeira dose, e que se atenuou ao longo de poucos dias a semanas. A resposta broncodilatadora ao agonista beta 2 permaneceu inalterada, apesar do tratamento contínuo com 
o beta-bloqueante. Os autores concluíram que os beta-bloqueantes cardioseletivos podem ser utilizados nos doentes com doença reativa das vias aéreas.

Em 2003, uma revisão sobre a segurança dos beta-bloqueantes nos doentes com insuficiência cardíaca e doença obstrutiva das vias aéreas concomitante concluiu que, como ocorreu na insuficiência cardíaca em que se registou inversão de paradigma, passando os beta-bloqueantes de contraindicados a indicados, os beta-bloqueantes cardioseletivos na asma poderão apresentar-se como um modo de tratamento das doenças obstrutivas das vias aéreas. ${ }^{49}$ Os resultados demonstraram que os beta-bloqueantes cardioseletivos não se associaram ao aumento dos sintomas respiratórios ou ao uso de inalador de alívio em doentes com DPOC ou doença reativa das vias aéreas e responderam aos broncodilatadores quando comparados com placebo.

Em 2011, uma meta-análise da Cochrane Library extraiu informações de 29 ensaios randomizados controlados por placebo, relativamente ao uso de beta-bloqueantes cardioseletivos em doentes com doença das vias aéreas com reversibilidade. ${ }^{1}$ Os resultados mostraram que, comparado com placebo, a primeira dose do tratamento ativo produziu um pequeno decréscimo no FEV1 que não foi associado a efeitos respiratórios adversos. Depois de tratamento continuado por alguns dias a semanas não se registou diferença no FEV1, sintomas ou uso de inalador de alívio. Os beta-bloqueantes cardioseletivos dados em dose única ou por tratamento continuado foram associados a um aumento na resposta broncodilatadora a agonistas beta 2 , quando comparados com placebo.

Em 2014, uma revisão sistemática com meta-análise, que apenas avaliava o efeito da exposição aguda a beta-bloqueantes cardioseletivos, demonstrou que $o$ beta-bloqueio cardioseletivo agudo nas doses dadas causava diminuições estatisticamente significativas do FEV1 e aumento da frequência dos sintomas, embora não estatisticamente significativos..$^{50}$ Os achados sugeriam que, apesar dos efeitos do beta-bloqueio seletivo agudo serem relativamente pequenos, poderiam, ainda assim, causar eventos clinicamente significantes numa minoria de doentes suscetíveis que tinham um tónus colinérgico excessivo. A resposta ao agonista beta 2 depois do beta-bloqueio seletivo teria sido parcialmente atenuada relativamente ao placebo. Os autores abordaram também, num editorial desse mesmo ano, o facto dos beta-bloqueantes cardioseletivos poderem desencadear exacerbações em asmáticos se iniciados em doses altas, dado que estas doses podem levar a que a cardioseletividade do fármaco possa ser perdida. ${ }^{51}$

Na presente revisão sistemática constatou-se que a exposição a doses únicas de beta-bloqueantes cardioseletivos levou a diminuições do FEVl estatisticamente significativas em $53,6 \%$ do total das 28 exposições. No entanto, estas reduções do FEV1 foram ligeiras (média de $8,24 \%$ e 0,24 l). O reduzido número de estudos que avaliaram doses múltiplas (dois ensaios) e contínuas (dois ensaios) não permite extrair conclusões válidas pelo risco de viés que poderia advir dessas mesmas conclusões. As coortes, já com um número significativo de doentes, revelaram conclusões variáveis, com um dos estudos referindo que estes fármacos não levaram a um maior risco de exacerbações de asma, outro mostrando segurança do seu uso no perioperatório e, por fim, um outro mostrando maior risco de exacerbações de asma (idas à urgência e internamentos).

Relativamente ao uso de beta-bloqueantes em doentes com asma e enfarte agudo de miocárdio ou insuficiência cardíaca, os beta-bloqueantes podem ter benefícios que eventualmente superam os seus riscos. Um estudo com sobreviventes de enfarte de miocárdio, que incluiu um total de 201.752 doentes, entre os quais 45.633 com asma ou DPOC, mostrou uma redução significativa na mortalidade total nos que foram tratados com beta-bloqueantes comparado com aqueles que não tinham sido tratados com estes fármacos. ${ }^{52}$ Também outro estudo demonstrou que, em indivíduos com hiperreatividade das vias aéreas que desenvolveram tanto enfarte de miocárdio como insuficiência cardíaca, os benefícios conhecidos de começar a terapêutica beta-bloqueante podem superar os seus riscos. ${ }^{49}$

Relativamente aos beta-bloqueantes tópicos oculares, estes fármacos são indicados para o tratamento do glaucoma, reduzindo a pressão intraocular pela diminuição da produção de humor aquoso. ${ }^{53}$ Uma metaanálise recente mostrou que os beta-bloqueantes não seletivos em administração ocular afetaram significativamente a função pulmonar (ocorreu uma diminuição média do FEV1 de 10,9\%) e aumentaram a incidência de exacerbações moderadas com a exposição aguda (IRR=4,83, IC95\%, 1,56-14,94)..$^{55}$ 
Um estudo de 2017 refere que, devido ao risco potencial destes agentes desencadearem broncoconstrição e uma resposta insuficiente à terapia com broncodilatadores durante a exacerbação, o uso de beta-bloqueantes não seletivos tópicos oculares em doentes com asma é contraindicado, conclusão esta que vai ao encontro das atuais guidelines de doenças pulmonares e glaucoma. ${ }^{54}$

É reconhecido que existe uma falta de estudos referentes a beta-bloqueantes tópicos oculares, sendo que uma explicação plausível para a exposição tópica ser de maior risco que a oral poderá ser pelo facto de ocorrer uma rápida absorção do fármaco para a circulação sistémica, sem haver metabolismo de primeira passagem e que esta exposição tópica tem efeitos semelhantes à administração IV relativamente à ocupação dos adrenorecetores beta 2 e efeitos cardiopulmonares. ${ }^{50}$

Na presente revisão sistemática os estudos analisados sugerem que os beta-bloqueantes tópicos oculares usados na prática clínica têm risco de exacerbações graves da asma, aumento de sintomas respiratórios e também reduções no FEV1, havendo, contudo, novos agentes que se mostram mais seguros (como fármacos mais oculoseletivos).

Relativamente ao aparente paradoxo do uso de beta-bloqueantes como tratamento para a asma, ao longo dos anos foram surgindo estudos que começaram a questionar a contraindicação do uso de beta-bloqueantes na asma, muitos deles criando hipóteses de que até poderiam ser vantajosos como tratamento da asma. Num estudo de 2008, num modelo de asma murino, é sugerido que o tratamento com beta-bloqueantes tanto cardioseletivos como não seletivos, numa dose baixa e dado de forma crónica, pode reduzir a inflamação das vias aéreas. ${ }^{41}$ Num outro estudo, em doentes com asma ligeira, verificou-se que a administração de doses escaladas do beta-bloqueante não seletivo nadolol foi segura, tendo também relatado benefícios a nível da hiperreatividade das vias aéreas com o uso crónico deste agente. ${ }^{11}$ Num estudo de 2009 sugere-se que uma razão para este possível benefício terapêutico de beta-bloqueantes na asma seria a conhecida upregulation dos adrenorecetores beta 2 com doses crónicas de beta-bloqueantes, contrastando, assim, com a down-regulation dos mesmos recetores que pode ocorrer com a exposição crónica a agonistas beta $2{ }^{40}$ Os mesmos autores discutem a possibilidade de haver benefícios no uso de beta-bloqueantes de forma crónica em asmáticos, ao contrário dos efeitos deletérios de doses agudas, e mencionam, em jeito de comparação, que o uso dos beta-bloqueantes na insuficiência cardíaca também foi inicialmente contraindicado devido à diminuição da contratilidade do miocárdio induzida pela dose aguda (levando a risco de edema pulmonar), tendo sido depois verificado que as doses crónicas produziam efeitos benéficos tanto na mortalidade como na fração de ejeção. ${ }^{39}$

Como limitações desta revisão sistemática é importante referir a heterogeneidade nas intervenções realizadas em cada estudo analisado, cada um com a sua metodologia específica e muitos deles avaliando apenas um tipo em particular de beta-bloqueante e cada um com diferentes doses (desde subterapêuticas a terapêuticas, sendo que as primeiras podem não evidenciar os efeitos reais na administração clínica) e diferentes vias de administração. Outra das limitações deriva do facto de cada um dos estudos apresentar um tempo variável de seguimento dos doentes, desde dias a semanas e anos. A maioria dos estudos incluídos avaliou a exposição aguda ao beta-bloqueio, sendo bastante reduzido o número de estudos que avaliaram uma exposição crónica.

Por outro lado, a maioria dos doentes incluídos nos estudos era jovem (idade média de 43,5 anos), com asma ligeira a moderada, sendo escassos os estudos que avaliaram doentes com asma grave, não sendo pois possível aplicar os resultados a estes doentes. A terapêutica de controlo da asma foi uma variável que diferiu entre os participantes de cada estudo.

Relativamente aos pontos fortes do artigo, a questão a ser respondida tem relevância na prática clínica na medida em que muitas vezes surge a dúvida de prescrever, ou não, beta-bloqueante, e de que tipo, ao doente asmático com comorbilidades que têm indicação para esta terapêutica.

O tipo de estudos incluídos, coorte e ensaios clínicos randomizados, por serem considerados de grande validade em termos de evidência clínica, constituiu um fator a favor deste artigo.

Em relação às implicações para a investigação, o facto dos beta bloqueantes não seletivos terem sido anteriormente associados com exacerbações de asma e 
mesmo a casos de morte, provavelmente levaram a um receio no desenvolvimento de estudos com estes fármacos, especialmente no que diz respeito ao seu uso em doentes com doença mais grave e com o uso de doses mais altas, sendo talvez por este motivo que sejam escassos os estudos existentes com estes agentes.

Sugere-se a continuidade de estudos referentes ao potencial efeito terapêutico dos beta-bloqueantes na asma para um melhor conhecimento da evidência e possível aplicação futura destes fármacos em doentes asmáticos com o objetivo de tratar a sua doença pulmonar.

Para concluir, os doentes com asma ligeira a moderada poderão, se indicado, ser medicados com beta-bloqueante, sempre com a vigilância do doente e com monitorização da função pulmonar e de sintomatologia que possa surgir de novo, particularmente nas fases iniciais do tratamento. Relativamente à cardioseletividade do agente escolhido não foi possível demonstrar na presente revisão sistemática uma preferência para agentes cardioseletivos ou não seletivos, o número de ensaios e exposições a beta-bloqueantes não seletivos foi bastante menor, pelo que não é lícito comparar e concluir sem um possível viés inerente.

Em relação à administração de beta-bloqueantes tópicos oculares em asmáticos, na presente revisão chama-se a atenção para o facto de que este tipo de fármacos pode ter efeitos prejudiciais, reduzindo o FEV1 e causando exacerbações de asma, havendo, contudo, novos agentes, mais oculoseletivos, que se revelam com maior margem de segurança que outros.

Já quanto à interessante perspetiva dos beta-bloqueantes poderem vir a estar indicados como terapêutica da própria asma, os estudos ainda são insuficientes para permitir uma recomendação a este nível.

\section{REFERÊNCIAS BIBLIOGRÁFICAS}

1. Salpeter S, Ormiston T, Salpeter E. Cardioselective beta-blockers for reversible airway disease. Cochrane Database Syst Rev. 2011;(4): CD002992.

2. Bortolotto LA, Consolim-Colombo FM. Betabloqueadores adrenérgicos [Adrenergic betablockers]. Rev Bras Hipertens. 2009;16(4):215-20. Portuguese

3. López-Sendón J, Swedberg K, McMurray J, Tamargo J, Maggioni AP, Dargie $\mathrm{H}$, et al. Expert consensus document on b-adrenergic receptor blockers. Eur Heart J. 2004;25(15):1341-62.

4. Ponikowski P, Voors AA, Anker SD, Bueno H, Cleland JG, Coats AJ, et al. 2016 ESC Guidelines for the diagnosis and treatment of acute and chro- nic heart failure: The Task Force for the diagnosis and treatment of acute and chronic heart failure of the European Society of Cardiology (ESC)Developed with the special contribution of the Heart Failure Association (HFA) of the ESC. Eur Heart J. 2016;37(27):2129-200.

5. Liberati A, Altman DG, Tetzlaff J, Mulrow C, Gøtzsche PC, loannidis JP, et al. The PRISMA statement for reporting systematic reviews and meta-analyses of studies that evaluate healthcare interventions: explanation and elaboration. BMJ. 2009;339:b2700.

6. Morales DR, Lipworth BJ, Donnan PT, Jackson C, Guthrie B. Respiratory effect of betablockers in people with asthma and cardiovascular disease: population based nested case control study. BMC Med. 2017;15(1):18.

7. Short PM, Anderson WJ, Williamson PA, Lipworth BJ. Effects of intravenous and oral B-blockade in persistent asthmatics controlled on inhaled corticosteroids. Heart. 2014;100(3):219-23.

8. Morales DR, Guthrie B, Lipworth BJ, Donnan PT, Jackson C. Prescribing of $\beta$-adrenoceptor antagonists in asthma: an observational study. Thorax. 2011;66(6):502-7.

9. Kaiserman I, Fendyur A, Vinker S. Topical beta-blockers in asthmatic patients: is it safe? Curr Eye Res. 2009;34(7):517-22.

10. Yamakage M, Iwasaki S, Jeong SW, Satoh JI, Namiki A. Beta-1 selective adrenergic antagonist landiolol and esmolol can be safely used in patients with airway hyperreactivity. Heart Lung. 2009;38(1):48-55.

11. Hanania NA, Singh S, El-Wali R, Flashner M, Franklin AE, Garner WJ, et al. The safety and effects of the beta-blocker, nadolol, in mild asthma: an open-label pilot study. Pulm Pharmacol Ther. 2008;21(1):134-41.

12. Brooks TW, Creekmore FM, Young DC, Asche CV, Oberg B, Samuelson WM. Rates of hospitalizations and emergency department visits in patients with asthma and chronic obstructive pulmonary disease taking beta-blockers. Pharmacotherapy. 2007;27(5):684-90.

13. Chen J, Radford MJ, Wang Y, Marciniak TA, Krumholz HM. Effectiveness of beta-blocker therapy after acute myocardial infarction in elderly patients with chronic obstructive pulmonary disease or asthma. J Am Coll Cardiol. 2001;37(7):1950-6.

14. Cazzola M, Noschese P, D'Amato M, D'Amato G. Comparison of the effects of single oral doses of nebivolol and celiprolol on airways in patients with mild asthma. Chest. 2000;118(5):1322-6.

15. Devereux G, Fishwick K, Aiken TC, Bourke SJ, Hendrick DJ. Adverse effects of a single dose of $(+)$-sotalol in patients with mild stable asthma. Br J Clin Pharmacol. 1998;46(1):79-82.

16. Bauer KG, Brunner-Ferber F, Distlerath LM, Lippa EA, Binkowitz et al. Assessment of bronchial effects following topical administration of butylamino-phenoxy-propanol-acetate, an oculoselective beta-adrenoceptor blocker in asthmatic subjects. Br J Clin Pharmacol. 1992;34(2): 122-9.

17. Pujet JC, Dubreuil C, Fleury B, Provendier O, Abella ML. Effects of celiprolol, a cardioselective beta-blocker, on respiratory function in asthmatic patients. Eur Respir J. 1992;5(2):196-200.

18. Hedner J, Ullman A, Lemne C, Svedmyr N. Effects of dilevalol, a betaadrenoceptor antagonist with intrinsic sympathetic activity in asthmatic patient. Pulm Pharmacol. 1989;2(3):155-9.

19. Van Zyl Al, Jennings AA, Bateman ED, Opie LH. Comparison of respiratory effects of two cardioselective beta-blockers, celiprolol and atenolol, in asthmatics with mild to moderate hypertension. Chest. 1989; 95(1):209-13. 
20. Chodosh S, Tuck J, Blasucci DJ. The effects of dilevalol, metoprolol, and placebo on ventilatory function in asthmatics. J Cardiovasc Pharmacol. 1988;11 Suppl 2:S18-24.

21. Richards R, Tattersfield AE. Comparison of the airway response to eye drops of timolol and its isomer L-714,465 in asthmatic subjects. Br J Clin Pharmacol. 1987;24(4):485-91.

22. Groth S, Tønnesen P, Asted M, Dirksen H, Sørensen PG. Assessment of the relative safety of the beta-blockers ICI 141,292 and atenolol in patients with bronchial asthma. Eur J Clin Pharmacol. 1986;30(6):653-8.

23. Philip-Joet F, Saadjian A, Bruguerolle B, Arnaud A. Comparative study of the respiratory effects of two beta 1-selective blocking agents atenolol and bevantolol in asthmatic patients. Eur J Clin Pharmacol. 1986;30(1):13-6.

24. Lammers JW, Müller ME, Folgering HT, van Herwaarden CL. A comparative study on the ventilatory and haemodynamic effects of xamoterol and atenolol in asthmatic patient. Br J Clin Pharmacol. 1986;22(5): 595-602.

25. Doshan HD, Brown R, Applin WJ, Kapoor M, Caruso FS. Effects of high doses of celiprolol in asthmatic patients. J Cardiovasc Pharmacol. 1986;8 Suppl 4:S109-11.

26. George RB, Light RW, Hudson LD, Conrad SA, Chetty K, Manocha K, et al. Comparison of the effects of labetalol and hydrochlorothiazide on the ventilatory function of hypertensive patients with asthma and propranolol sensitivity. Chest. 1985; 88(6):815-8.

27. Lammers JW, Folgering $H T$, van Herwaarden CL. Ventilatory effects of atenolol and bevantolol in asthma. Clin Pharmacol Ther. 1985;38(4): 428-33.

28. Clague HW, Ahmad D, Carruthers SG. Influence of cardioselectivity and respiratory disease on pulmonary responsiveness to beta-blockade. Eur J Clin Pharmacol. 1984;27(5):517-23.

29. Lammers JW, Folgering HT, van Herwaarden CL. Ventilatory effects of beta 1-receptor-selective blockade with bisoprolol and metoprolol in asthmatic patients. Eur J Clin Pharmacol. 1984;27(2):141-5.

30. Patakas D, Argiropoulou V, Louridas G, Tsara V. Beta-blockers in bronchial asthma: effect of propranolol and pindolol on large and small airways. Thorax. 1983;38(2):108-12.

31. Jackson SH, Beevers DG. Comparison of the effects of single doses of atenolol and labetalol on airways obstruction in patients with hypertension and asthma. Br J Clin Pharmacol. 1983;15(5):553-6.

32. Lawrence DS, Sahay JN, Chatterjee SS, Cruickshank JM. Asthma and beta-blockers. Eur J Clin Pharmacol. 1982;22(6):501-9.

33. Ruffin RE, Mclntyre EL, Latimer KM, Ward HE, Crockett AJ, Alpers JH. Assessment of beta-adrenoceptor antagonists in asthmatic patients. $\mathrm{Br}$ J Clin Pharmacol. 1982;13(Suppl 2):325S-35S.

34. Dorow P. Influence of intrinsic sympathomimetic activity (ISA) during beta-adrenoceptor blockade in asthmatics. Br J Clin Pharmacol. 1982;13 Suppl 2:321S-3S.

35. Sue DY, Hansen JE, Wasserman K. Beta-adrenergic blockade with pindolol (LB-46) in mild to moderate asthma. Chest. 1981;80(5):537-42.

36. Loth DW, Brusselle GG, Lahousse L, Hofman A, Leufkens HG, Stricker $\mathrm{BH}$. $\beta$-Adrenoceptor blockers and pulmonary function in the general population: the Rotterdam study. Br J Clin Pharmacol. 2014;77(1):190-200.

37. Shaukat A, Kazmi S, Shaheen R. In vitro response of tracheal smooth muscle from hyperresponsive guinea pigs to celiprolol. J Ayub Med Coll
Abbottabad. 2012;24(3-4):85-6.

38. Short PM, Williamson PA, Lipworth BJ. Sensitivity of impulse oscillometry and spirometry in beta-blocker induced bronchoconstriction and beta-agonist bronchodilatation in asthma. Ann Allergy Asthma Immunol. 2012;109(6):412-5.

39. Lipworth BJ, Williamson PA. Think the impossible: beta-blockers for treating asthma. Clin Sci. 2009;118(2):115-20.

40. Lipworth BJ, Williamson PA. $\beta$ blockers for asthma: a double-edged sword. Lancet. 2009;373(9658):104-5.

41. Nguyen LP, Omoluabi O, Parra S, Frieske JM, Clement C, Ammar-Aouchiche Z, et al. Chronic exposure to beta-blockers attenuates inflammation and mucin content in a murine asthma model. Am J Respir Cell Mol Biol. 2008;38(3):256-62.

42. Boskabady MH, Kiani S, Aslani MR. Tracheal responsiveness to both isoprenaline and beta2-adrenoreceptor blockade by propranolol in cigarette smoke exposed and sensitized guinea pigs. Respirology. 2006;11 (5):572-8.

43. Oosterhoff Y, Koëter GH, Postma DS. Effects of propranolol inhalation on the diurnal increase in FEV1 and on propranolol airways responsiveness in atopic subjects with asthma. Thorax. 1995;50(9):937-40.

44. McNeill RS. Effect of a beta-adrenergic-blocking agent, propranolol, on asthmatics. Lancet. 1964;2(7369):1101-2.

45. Zaid G, Beall GN. Bronchial response to beta-adrenergic blockade. N Engl J Med. 1966;275(11):580-4.

46. Anderson EG, Calcraft B, Jariwalla AG, Al-Zaibak M. Persistent asthma after treatment with beta-blocking agents. $\mathrm{Br}$ J Dis Chest. 1979;73(4): 407-8.

47. Raine JM, Palazzo MG, Kerr JH, Sleight P. Near-fatal bronchospasm after oral nadolol in a young asthmatic and response to ventilation with halothane. Br Med J. 1981;282(6263):548-9.

48. Salpeter SR, Ormiston TM, Salpeter EE. Cardioselective beta-blockers in patients with reactive airway disease: a meta-analysis. Ann Intern Med. 2002;137(9):715-25.

49. Ormiston TM, Salpeter SR. Beta-blocker use in patients with congestive heart failure and concomitant obstructive airway disease: moving from myth to evidence-based practice. Heart Fail Monit. 2003;4(2):4554.

50. Morales DR, Jackson C, Lipworth BJ, Donnan PT, Guthrie B. Adverse respiratory effect of acute $\beta$-blocker exposure in asthma: a systematic review and meta-analysis of randomized controlled trials. Chest. 2014; 145(4):779-86.

51. Morales D. Initiating beta-blockers in patients with asthma. Prescriber. 2014;25(19):9-10.

52. Gottlieb SS, McCarter RJ, Vogel RA. Effect of beta-blockade on mortality among high-risk and low-risk patients after myocardial infarction. N Engl J Med. 1998;339(8):489-97.

53. Arboe B, Ulrik CS. Beta-blockers: friend or foe in asthma? Int J Gen Med. 2013;6:549-55.

54. Kuipers E, Wensing M, De Smet PA, Teichert M. Considerations of prescribers and pharmacists for the use of non-selective $\beta$-blockers in asthma and COPD patients: an explorative study. J Eval Clin Pract. 2018;24(2):396-402.

55. Morales DR, Dreischulte T, Lipworth BJ, Donnan PT, Jackson C, Guthrie B. Respiratory effect of beta-blocker eye drops in asthma: population- 
based study and meta-analysis of clinical trials. $\mathrm{Br} J$ Clin Pharmacol. 2016;82(3):814-22.

\section{CONFLITO DE INTERESSES}

Os autores declaram não possuir quaisquer conflitos de interesse.

FINANCIAMENTO DO ESTUDO

Os autores declaram não ter recebido qualquer financiamento do estudo.
ENDEREÇO PARA CORRESPONDÊNCIA

Mariana Baltazar Coelho

E-mail: marianamrbaltazarcoelho@gmail.com

https://orcid.org/0000-0002-8403-9476

Recebido em 27-04-2020

Aceite para publicação em 19-11-2020

\section{ABSTRACT}

\section{BETA-BLOCKERS AND ASTHMA}

Background: Asthmatic patients may have some conditions for which the use of beta-blockers is required. Therapy with these drugs is used in several diseases and it is widely used in clinical practice, especially in primary care. This means that it is important to clarify possible contraindications to its use in subjects with asthma.

Objectives: Evaluating the effects of cardioselective and non-selective beta-blockers in asthmatic patients: changes in the pulmonary function tests; acute exacerbations of the disease and its symptomatology; the frequency that they need to use rescue drugs; the need to use an emergency department and frequency of hospitalizations related to the adverse effects caused by these drugs.

Methods: This systematic review included articles searched in PubMed electronic database. The search term used was: asthma AND beta-blockers.

Results: There were 151 articles identified, from which 49 were selected after reading the titles and abstracts. Of these, after reading the complete article and applying the inclusion criteria, 30 articles were selected. Atenolol and propranolol were the most frequently studied beta-blockers. Two randomized clinical trials and one cohort were about topical beta-blockers; 22 randomized clinical trials studied oral or intravenous beta-blockers. Five cohorts were also discussed.

Conclusion: Beta-blockers in mild to moderate asthma can be used but always with monitoring of pulmonary function and symptoms of the patient. We could not demonstrate a preference for drug selectivity. Topical ocular beta-blockers in asthma can have detrimental effects by reducing FEV 1 and increasing asthma exacerbations. We cannot recommend the use of betablockers as a treatment for asthma since we still have few studies on this topic.

Keywords: Asthma; Beta-blocker; FEV1; Bronchoconstriction; Wheezing. 